\title{
Research Paper \\ Death Anxiety and Its Relationship with Social Support and Adherence to Religion in the Elderly
}

\author{
*Seyyed Abolghasem Mehri Nejad', Leyly Ramezan Saatchi', Sayeh Paydar ${ }^{3}$ \\ 1. Department of Psychology, Faculty of Education \& Psychology, Alzahra University, Tehran, Iran. \\ 2. Department of Clinical Psychology, Faculty of Education \& Psychology, Shahid Chamran University of Ahvaz, Ahvaz, Iran. \\ 3. Department of Psychology, Faculty of Humanities, Shahrood Branch, Islamic Azad University, Shahrood, Iran.
}

Received: 13 Jun. 2016

Accepted: 18 Oct. 2016

Key words:

Aged, Death anxiety,

Social support, Ad-

herence to religion
Cltation: Mehri Nejad SA, Ramezan Saatchi L, Paydar S. [Death Anxiety and Its Relationship with Social Support and Adherence to Religion in the Elderly (Persian)]. Iranian Journal of Ageing. 2017; 11(4):494-503. http://dx.doi.org/10.21859/sija-1104494

doi: : http://dx.doi.org/10.21859/sija-1104494

\begin{abstract}
A B S TRACT
Objectives Aging is a biological process, experienced by all living things, including humans. The most important factor in this period is the death anxiety. This study aimed to determine death anxiety and its relationship with social support and adherence to religion in unmarried and married men and women.

Methods \& Materials In this study, causal-comparative and correlation methods were used. The study was conducted on 376 people; 190 men and 186 women (married and unmarried). The participants were selected from high school graduates from districts 2, 3, 5, 6, and 22 . They were 60 and 75 years, and selected by using convenience sampling. Death anxiety scales 27 -item form, 19-item form for social support questionnaire and 26 -item form for religious attitudes were used to measure study variables. Data were analyzed by 1-way ANOVA and the Pearson tests using the SPSS 21.

Results The results of correlation analysis indicated significant and direct relationship between social support and the fear of dying $(P<0.05)$ and fear of death by others $(P<0.05)$, as well as with the overall death anxiety $(P<0.01)$. With regard to the relationship between adherence to religion and death anxiety components, only the relationship between faith and fear of the consequences of dying was significant $(P<0.01)$. The analysis of variance indicated a significant difference between unmarried and married men with regard to death anxiety. Unmarried men experienced more death anxiety $(P<0.01)$. The average death anxiety scores among women was more than that among men $(P<0.01)$, but there was no significant difference between unmarried and married women with regard to death anxiety.

Conclusion Increased social interaction, adherence to religion and marriage can decrease death anxiety and improve the mental health of the elderly, especially elderly women.
\end{abstract}




\title{
بررسى رابطه بين حمايت اجتماعى و پايبندى به مذهب با اضطراب مرتى در سالمندان
}

\author{
"سيدابوالقاسم مهرىنزןاد'، ليلى رمضانساعتجى"، سايه بايدار”

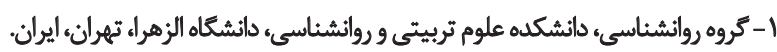

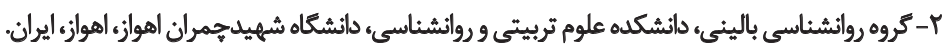

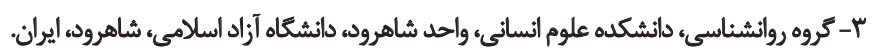

\begin{abstract}
حكSد

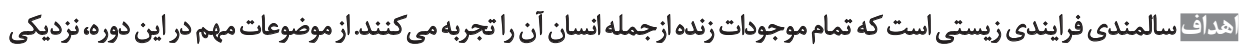

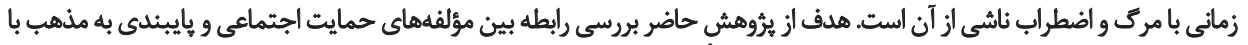

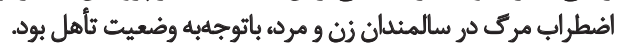

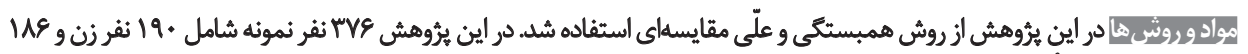

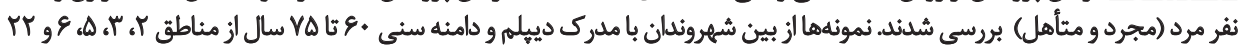

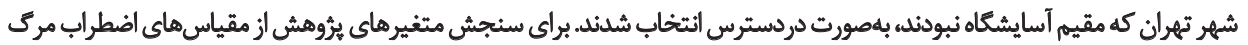

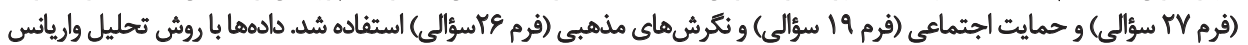

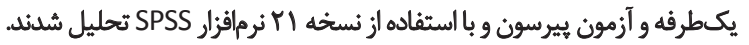

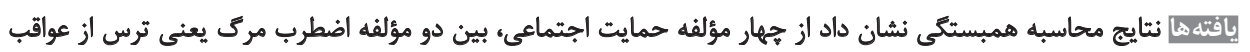

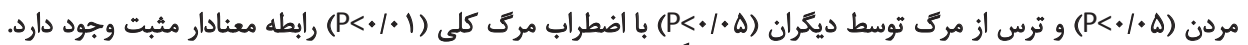

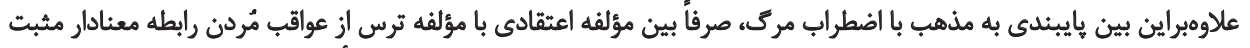

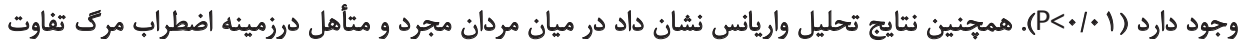

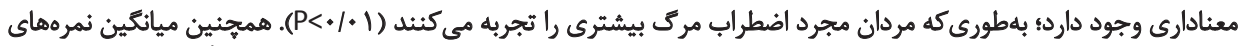

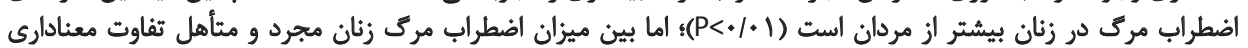
وجود ندارد.

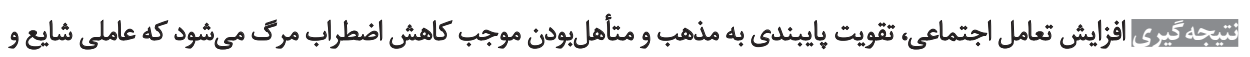

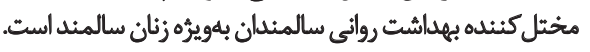

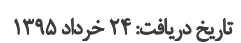

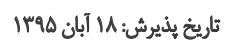

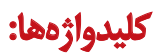

سالمندي، اضطراب مركى، حمايت اجتماعي، بايبندى بله مذهب

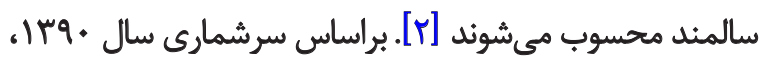

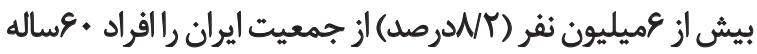

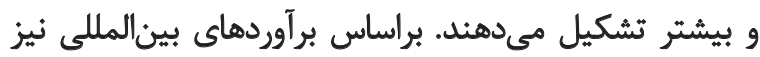

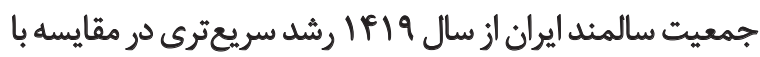

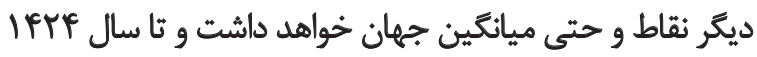

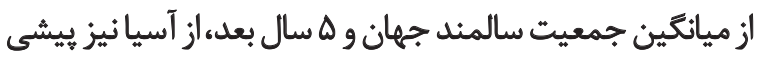
خواهد كرفت [ب].

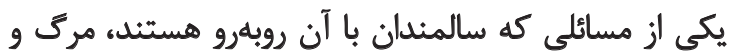

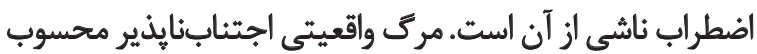

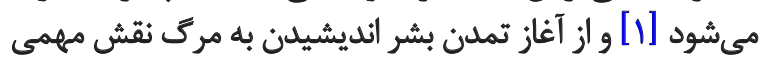

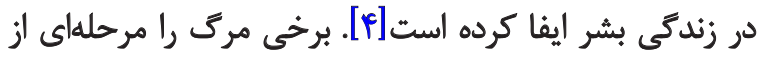

dodits سالمندى فرايندى زيستى است كه تمام موجودات زنده

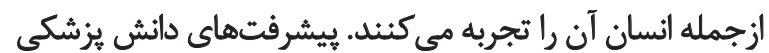

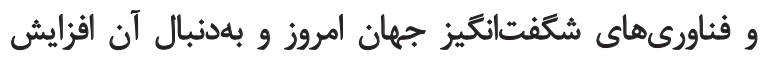

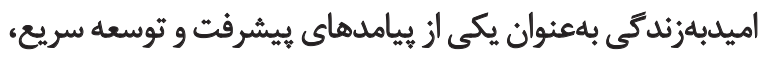

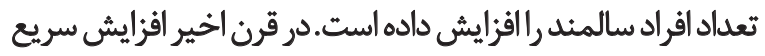

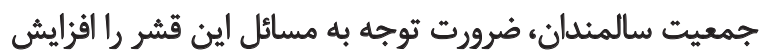

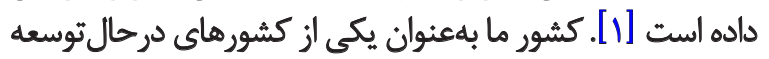

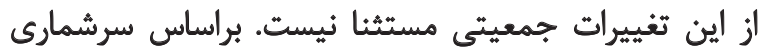

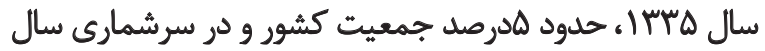

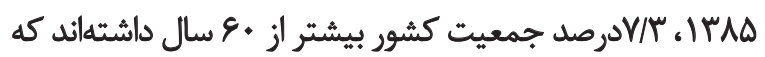


افراد شواهد محكمى براى اثبات اين قضيه هستند. علاوهبراين

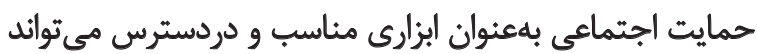

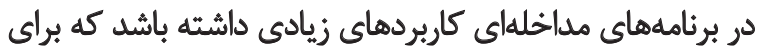

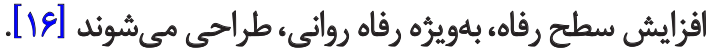
محققان بهطورمداوم دريافتند حمايتهاي اجتماعى سالمندان ماندان

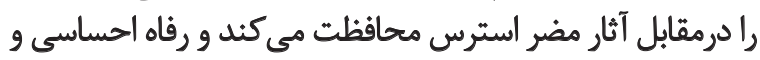

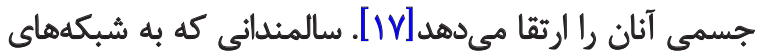

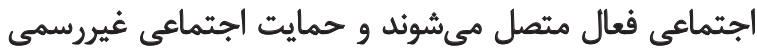

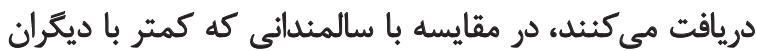

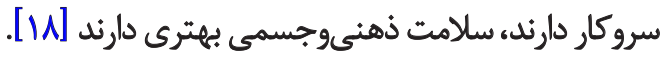

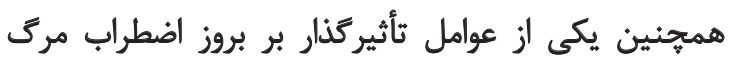

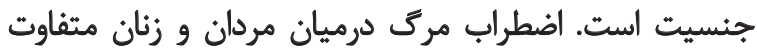

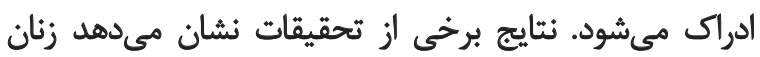

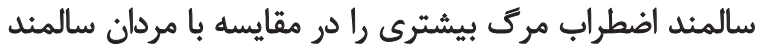

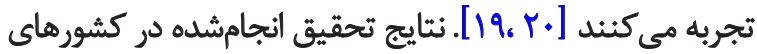

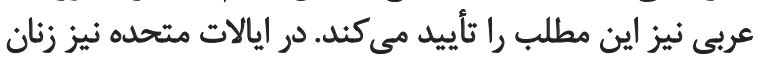

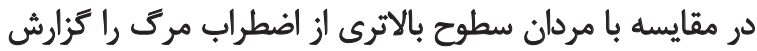

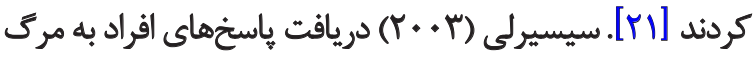

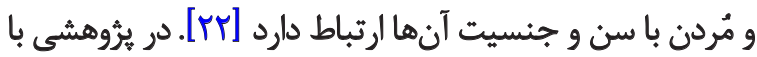

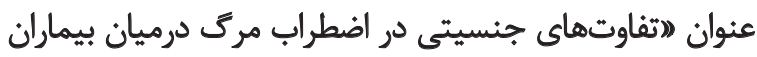

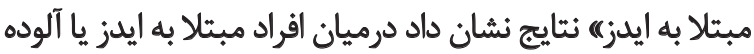

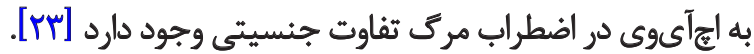
عامل ديخرى كه اضطراب مرى را را متفاوت مىسازد، وضعيث

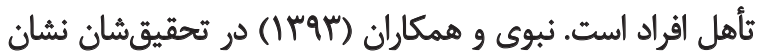
دادند سالمندان متأهل سلامت رواند

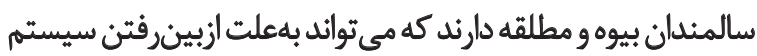

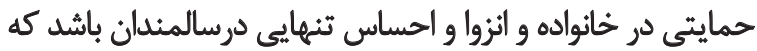

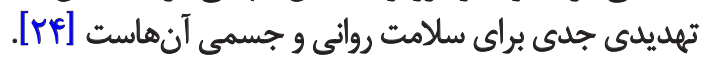

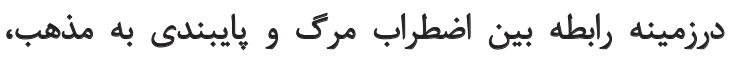

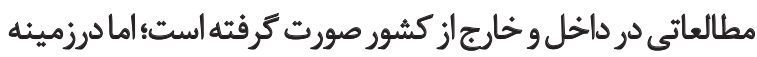

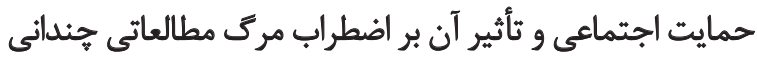

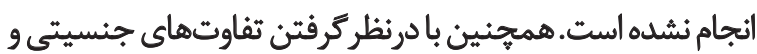

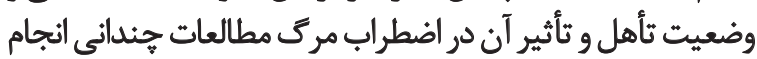

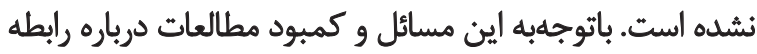

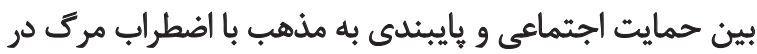

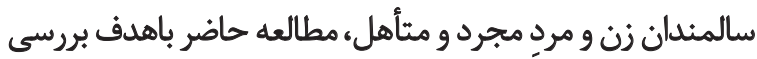

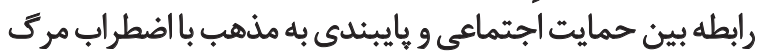

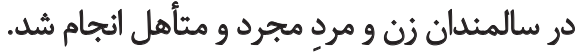

روش مطالعه

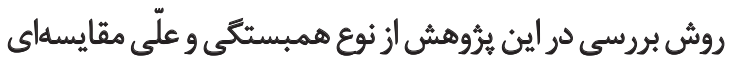

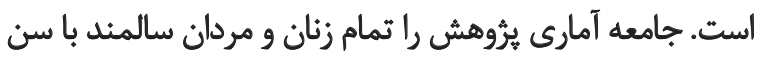

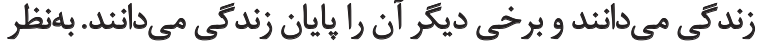

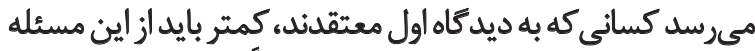

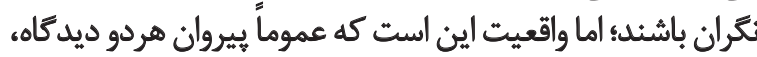

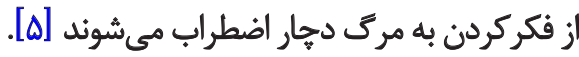

اضطراب مرك مفهومى جندبُعدى و تعريف آن امرى دشوار

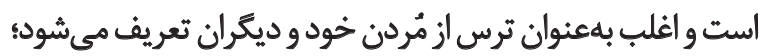

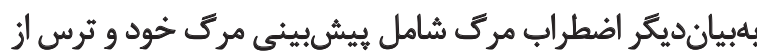

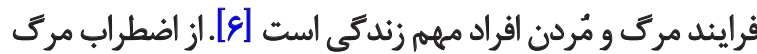

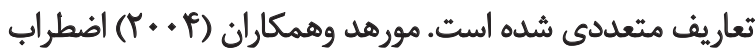

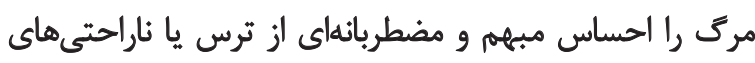

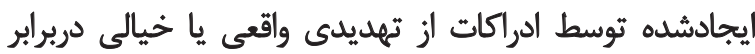

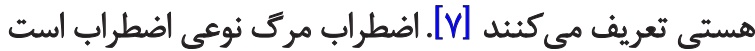

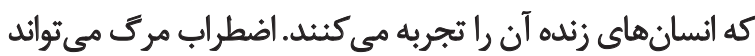

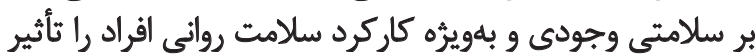

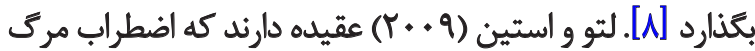

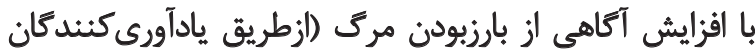

فانىبودن شخص) آغاز مي أشود [9]

مواردزيادى در كاهش يا افزايش اضطراب مرك مؤثر هستند كه إنها

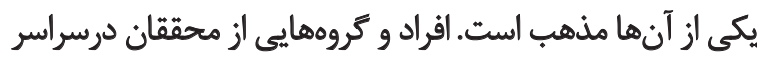

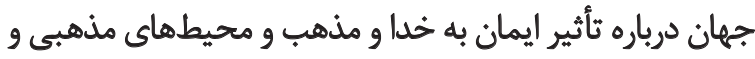

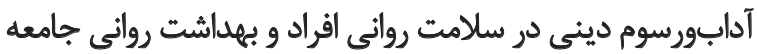

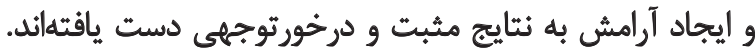

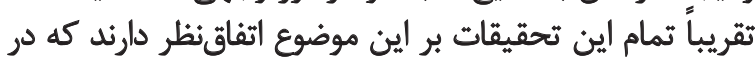

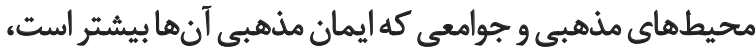

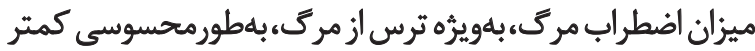

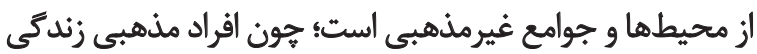

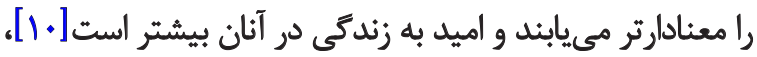
يس اضطراب مركى در آنان كمتر است.

در بررسى هايى كه روى زنان و مردانى انجام شد كه جهت كيري مئرى

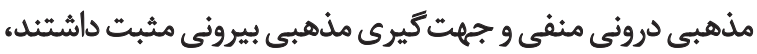

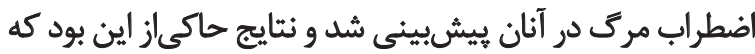

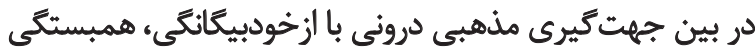

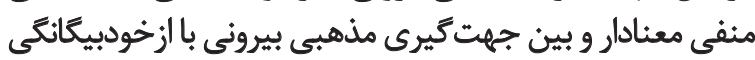

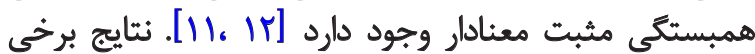

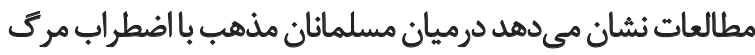

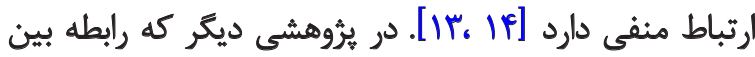

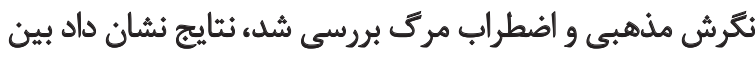

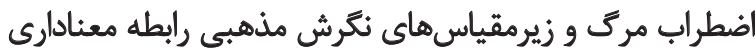

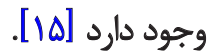

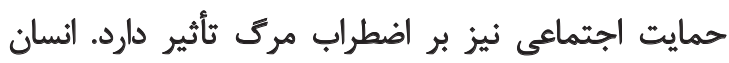

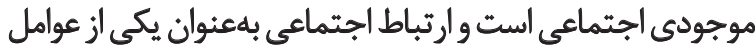

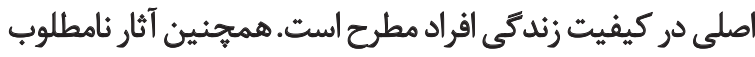

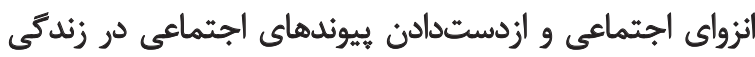




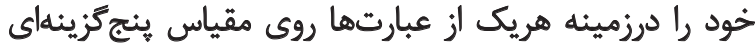

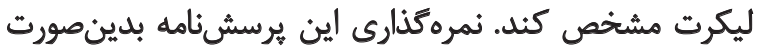

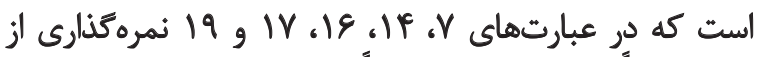

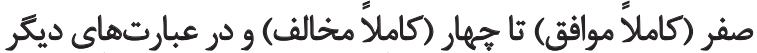

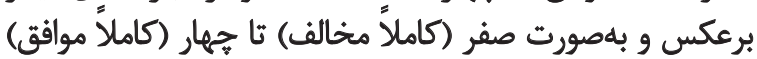

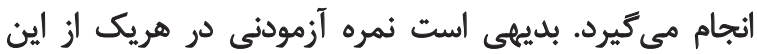

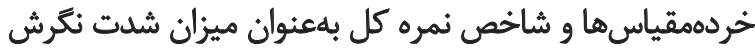

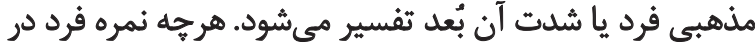

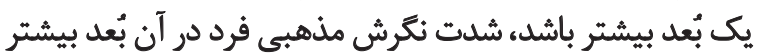

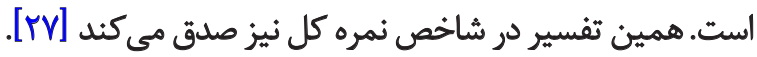
روايى صورى اين برسشنامه با نظرخواهى از تعدائي

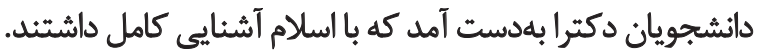

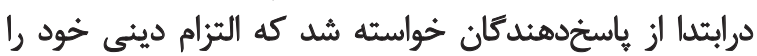

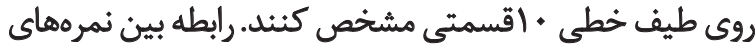

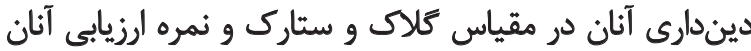

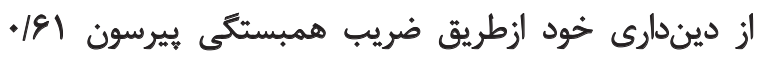

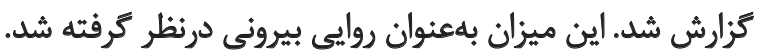

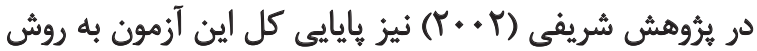

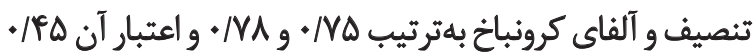

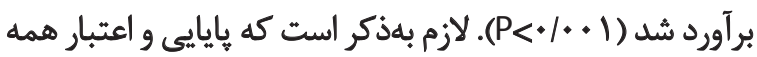

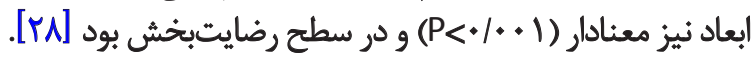

يافتهها

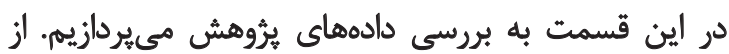

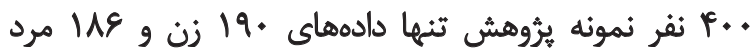

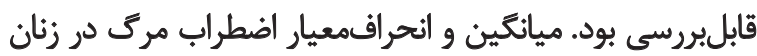

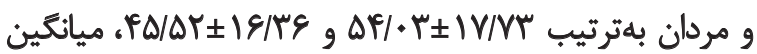

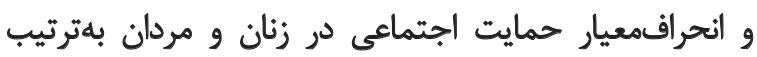
نكرش مذهب

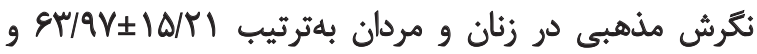

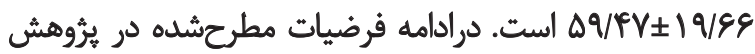

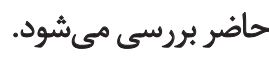

فرضيه اول: بين مؤلفههاى حمايت اجتماعى و اضطراب مركى

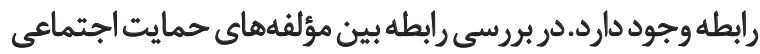

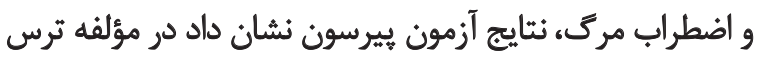

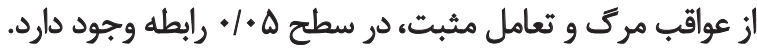

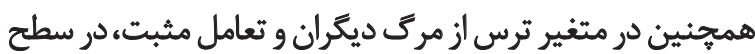

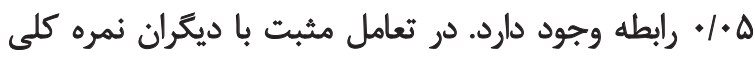

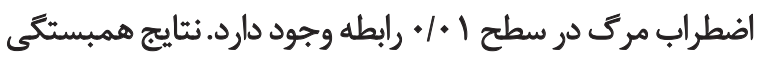

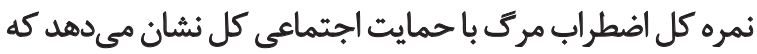

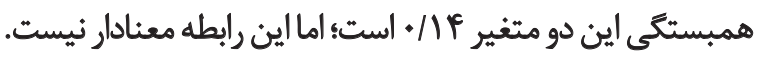

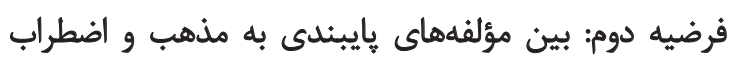

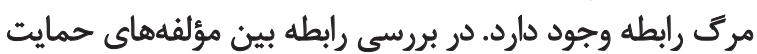

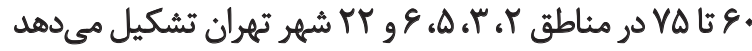

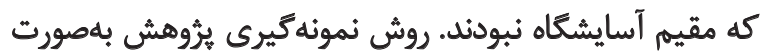

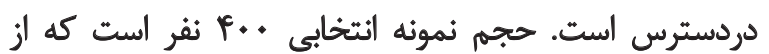

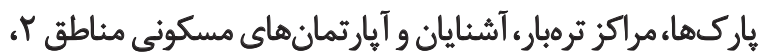

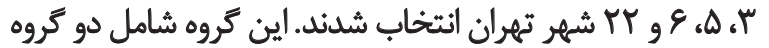

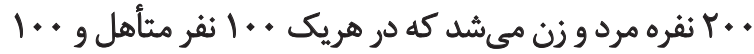

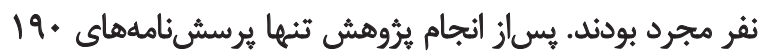

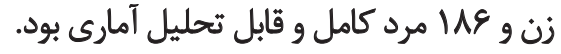
در اين يُروهش از سه يرسشنامه استفاده شد:

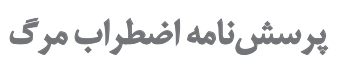

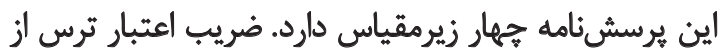

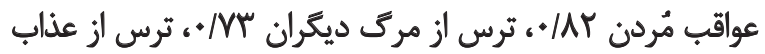

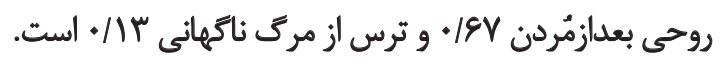

$$
\text { يرسش نامه حمايث إجتماعى }
$$

يرسشنامه حمايت اجتماعى شربورن و استوارت در سال 1991

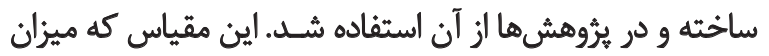

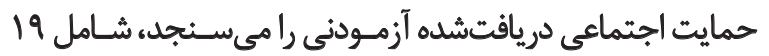

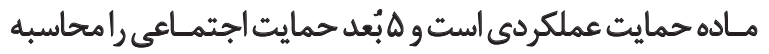

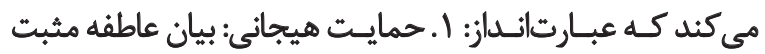

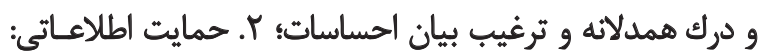

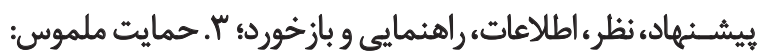

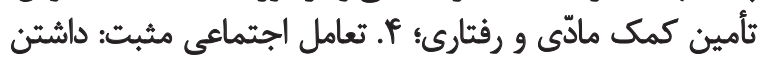

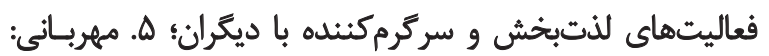

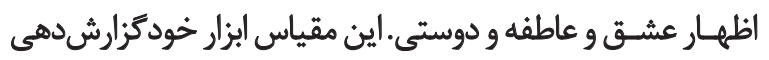

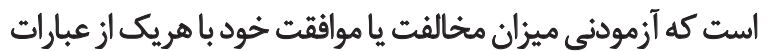

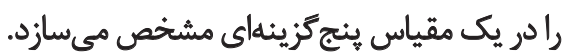
كمترين امتياز در اين آزمون 19 و و بيشترين امتياز هو 9 است.

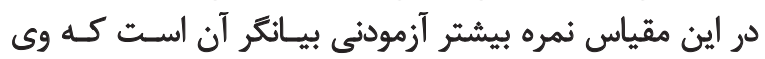

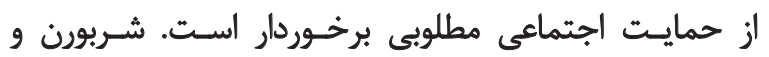

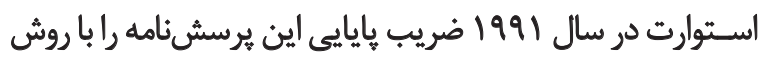

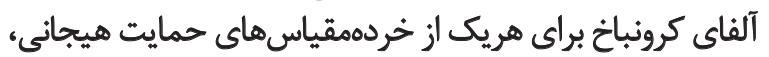

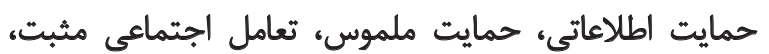

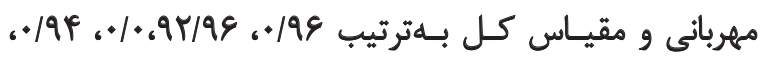

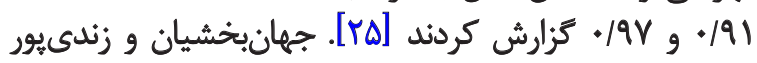

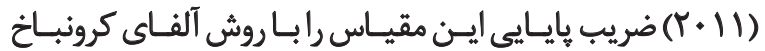

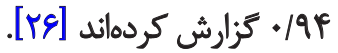

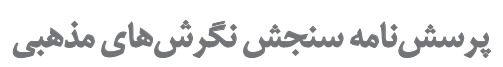

اين برسشنامه \&Y عبارت دارد كه جهار بُعد (زيرمقياس)

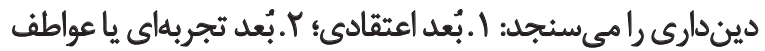

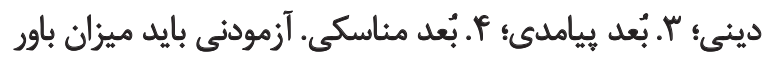


جدول ا. توزيع فراوانى شركت كنئدكان در يُروهش.

\begin{tabular}{|c|c|c|c|c|c|}
\hline درصد فراوانى & فراواثى & وضعيت تأهل & درصد فراوانى & فراوانى & جنسيت \\
\hline $\begin{array}{l}P V+\Delta \\
\Delta N q \Delta\end{array}$ & $\begin{array}{l}\text { va } \\
\text { irr }\end{array}$ & متأهل & $p q$ & 19. & زن \\
\hline $\begin{array}{l}r \text { r/AV } \\
\text { selIr }\end{array}$ & אז & مجأجرد & 0) & $w$ & هرد \\
\hline
\end{tabular}

记

جدول r. رابطه مؤلفههاى حمايت اجتماعى و مؤلفههاى اضطراب مركى.

\begin{tabular}{|c|c|c|c|c|c|c|}
\hline اضطراب مركى & ترسئمانمى ازمرى & ترو الز عذي بعداب & توسيكران مرى & ترس از عواقب & & \\
\hline$\cdot 1+M$ & $-.1 .+1$ & $1 . n$ &.$/ . r A$ & $-+1 \cdot+v$ & & \multirow{2}{*}{ حمايت عاطفى / اطلاعرسانى } \\
\hline$+/ M T$ & $+109+$ & $+/ 4+8$ & +1980 &.$/ q 4 \mathrm{~A}$ & معنادارى & \\
\hline & & $-+/ 14 \lambda$ & $+1+1 Y$ &.$/ 1 \cdot 4$ & ي ييرسون & \multirow{2}{*}{ حمايت ملموس } \\
\hline$\cdot / \mathrm{VA}$ & $\cdot / 4 \cdot 0$ & .1 .94 & - &.$/ M F$ & معنادارى & \\
\hline t/Mment &.$- / \cdot n$ & $-* / \cdot v$ & $. / \pi)^{*}$ & $* / \pi *_{*}^{*}$ & ييرسون & \multirow{2}{*}{ تعامل مثبت } \\
\hline.$\circ \mathrm{V}$ &. $\mid$. 91 & . & .1 .18 & .1 .14 & ت & \\
\hline .1 .90 & $-.1 \cdot r$ &.$- / 1 r$ &.. .4 & .1 .9$. & ييرسون & \multirow{2}{*}{ هـربانى } \\
\hline.$/ T A I$ & $\cdot \mid M^{\prime \prime}$ &.$/ 1 Y a$ &.$/ 9 w$ &.$/ \Delta \cdots$ & هعiادارى & \\
\hline . Nift & $-.1 \cdot \Delta$ & $-.11 \cdot 8$ & $.11 \cdot 9$ & - lirr & ييرسون & \multirow{2}{*}{ حمايت اجتماعي كل } \\
\hline.$/ 1 \cdot r$ & -/AYQ &.$/ 4 r q$ &.$/ K M$ & 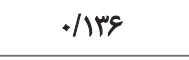 & تمعنادارى & \\
\hline
\end{tabular}

جدول r. رابطه مؤلفههاى بايبيندى به مذهب و مؤلفههاى اضطراب مركى.

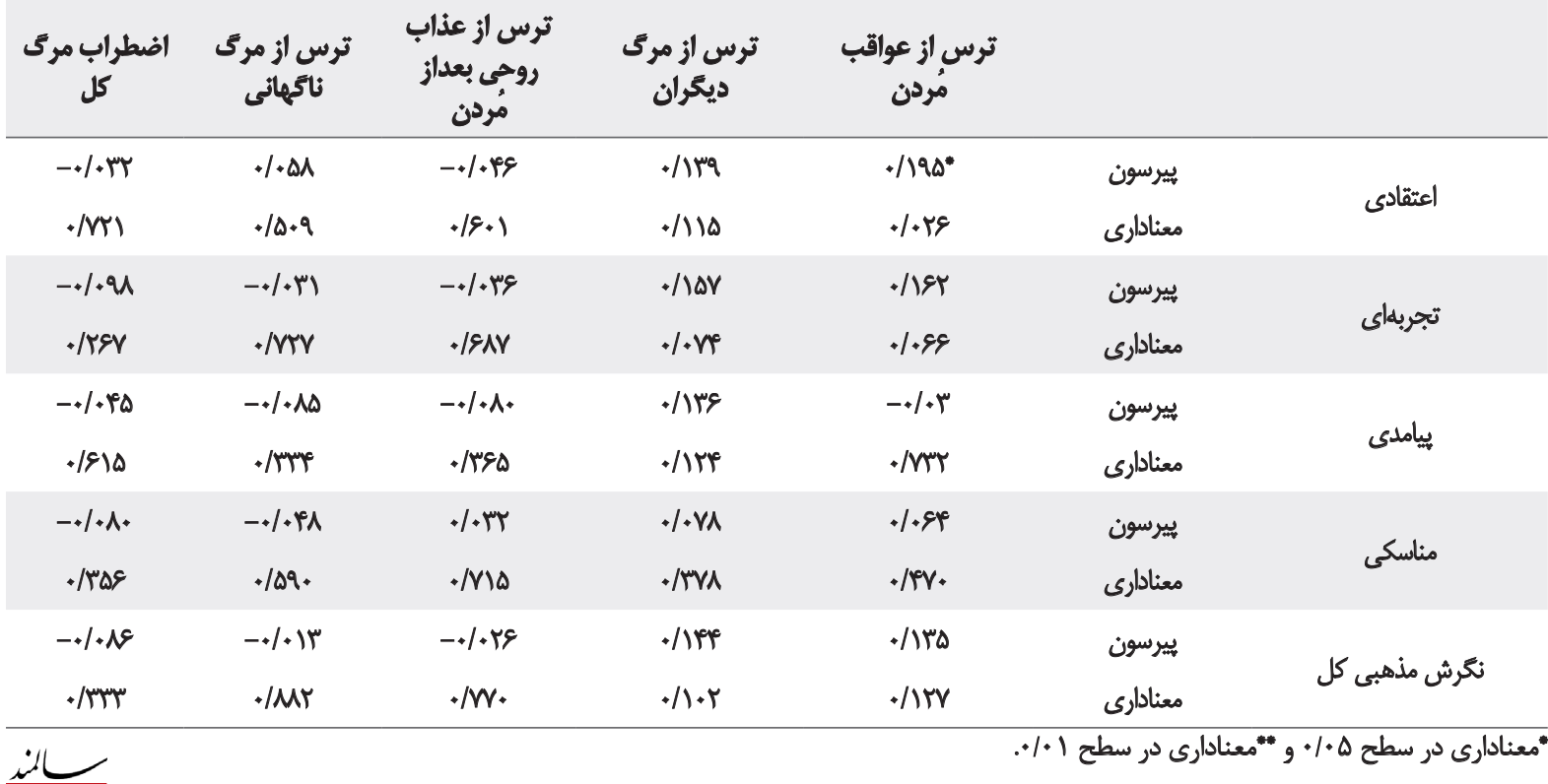


جدول P. مقايسه اضطراب مرك در بين زنان و مردان مجرد و مثأهل.

\begin{tabular}{|c|c|c|c|c|c|}
\hline معناداري & $\mathbf{F}$ & ورجه آزادى & & & \\
\hline \multirow{3}{*}{. $/ A T$} & \multirow{3}{*}{. I8AV } & 1 & rIV/FIr & بين كروهي & \multirow{3}{*}{ اضطراب مرى در زئان } \\
\hline & & $\Delta q$ & $19.1 \% / \Delta A Y$ & درون كروهى & \\
\hline & & 8. & IQYTR/YIY & جمع & \\
\hline \multirow{3}{*}{$.1 \cdot Y \varphi$} & \multirow{3}{*}{ ه/TTQ } & 1 & $\| r+\Delta / V \cdot A$ & يينكروهى & \multirow{3}{*}{ اضطراب مرى مر مر مردان } \\
\hline & & \&V & IETqV/TEA & درون كروهى & \\
\hline & & 81 & $|w \cdot r| \cdot v r$ & جمع & \\
\hline
\end{tabular}

L

بينفردى اهميت ويرّاي در رويكرد افراد به مرك دارد و ممكن است اضطراب مرك را كاهش دهيد.

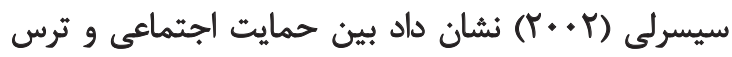

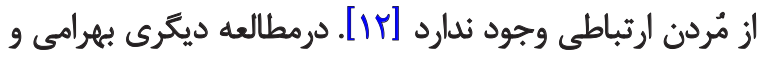

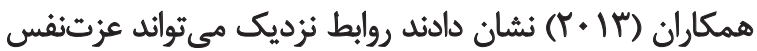

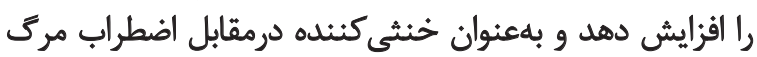

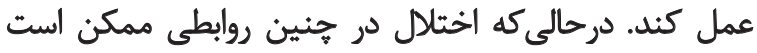

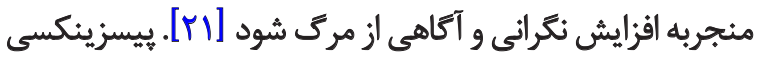

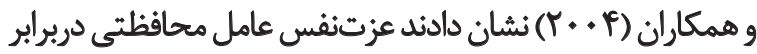

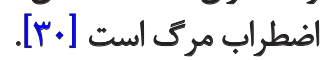

مطالعات متعددى نشان داده است كه سالمندان ساكن در

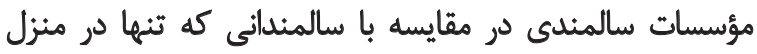

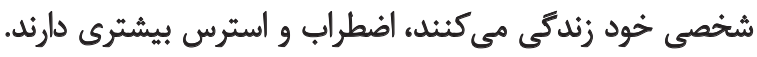

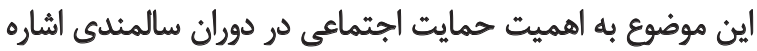

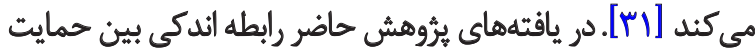

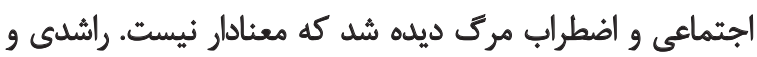

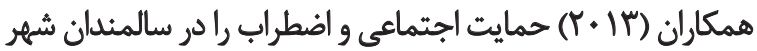

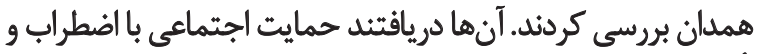

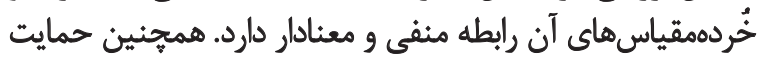

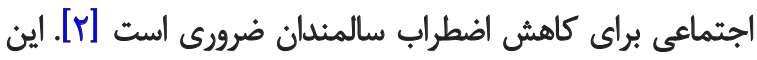

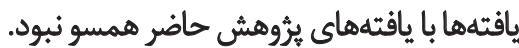

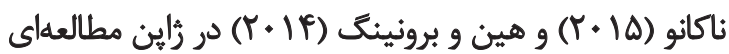

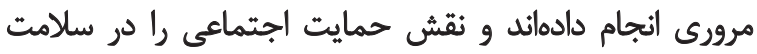

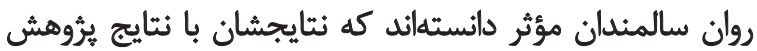

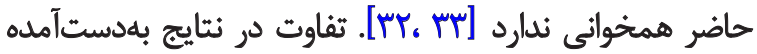

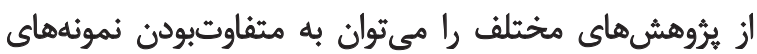

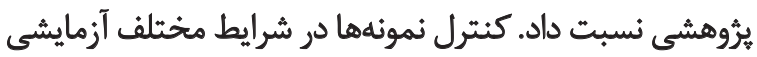

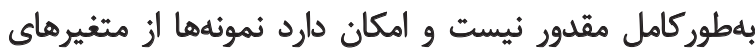
ديكرى تأثير بذيرفته باشند.

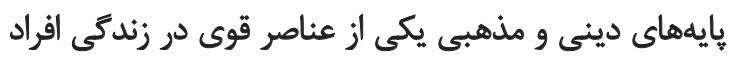
جامعه ايران است؛ بنابراين افراد نكُش شهاي خاصي درئ دربر دابر مسئله
اجتماعى و عايبندى به مذهب، نتايج آزمون ييرسون نشان داد

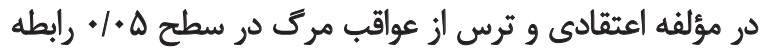

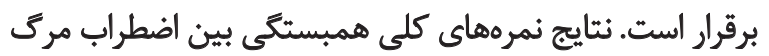

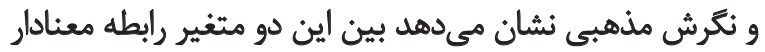

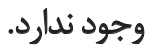
فرضيه سوم: اضطراب مرك در بين زنان و مردان هجرد و متأهل

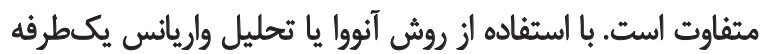

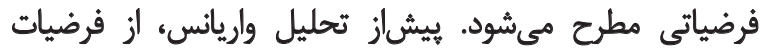

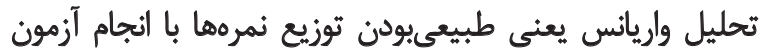

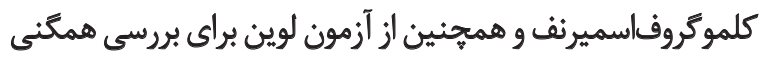

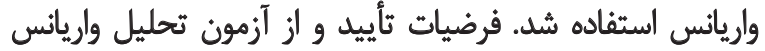

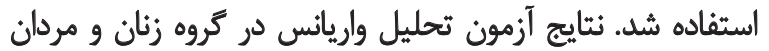

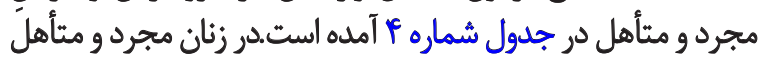

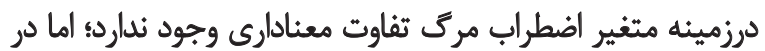
مردان تفاوت معنادارى وجود دارد و فرض صفر تأييد مي وشود.

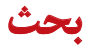
هدف از يُروهش حاضر بررسى رابطه ميان اضطراب هرك

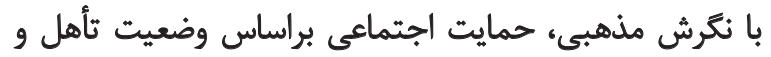

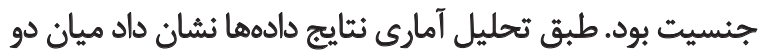

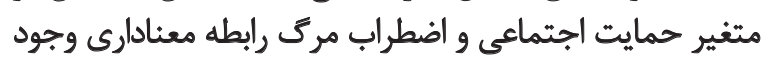

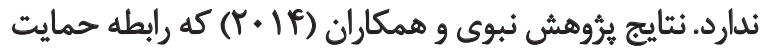

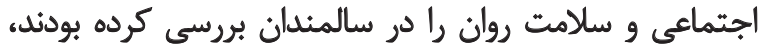

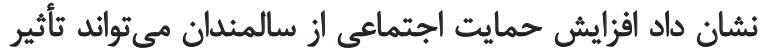

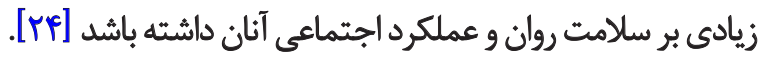

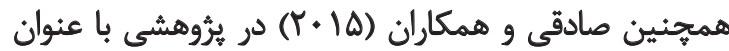

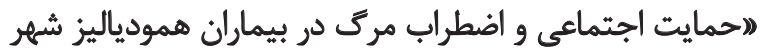

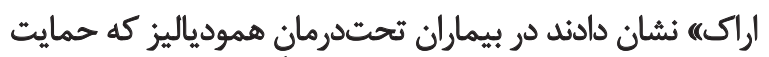

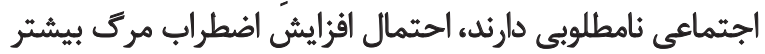

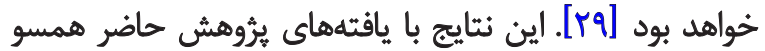

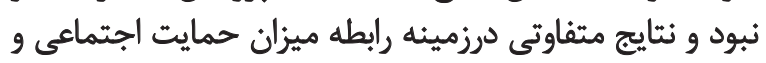
اضطراب مرك داشت. بهنظر ميرسد حمايت اجتماعى و تعاملات 
و مجرد بلصورت متفاوت درك ميش مود؛؛ اما اضطراب مركى در

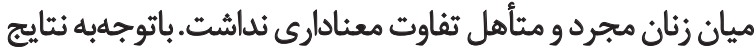

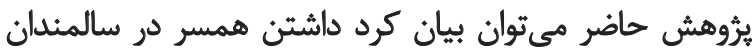

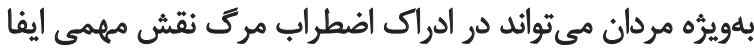

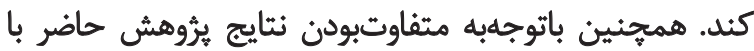

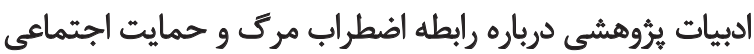

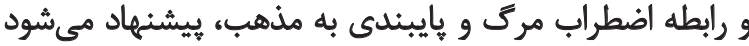
يروهشهاى بيشتر در اين زمينه انجام شود.

محدوديتها

اين يرؤهش همجون يُروهشهاى ديكر با محدوديتهايى

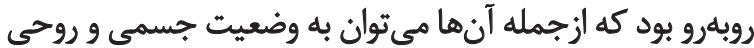

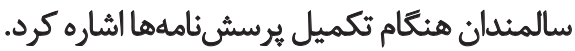

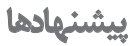

درنهايت بيشنهاد ميشود باتوجهبه اهميت مسئله سالمندى

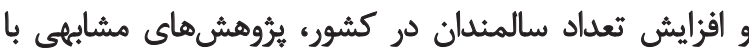

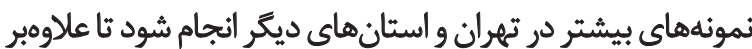

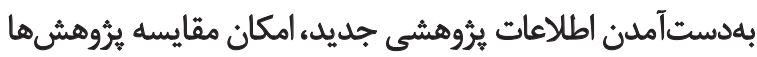

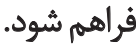

$$
\text { تشكر و قدردانى }
$$

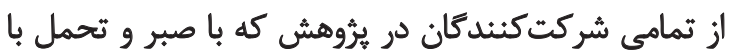

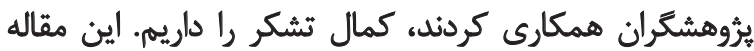
خامى مالى نداشته است.

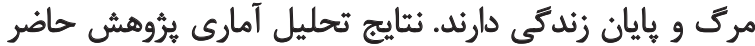

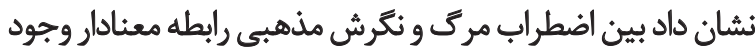

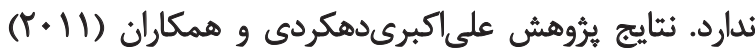

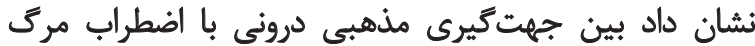

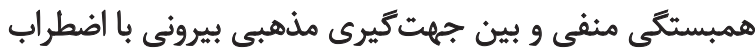

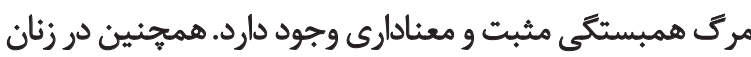

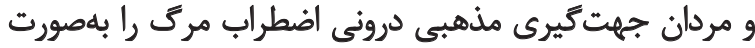

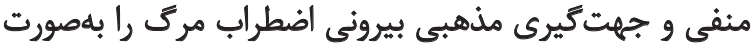

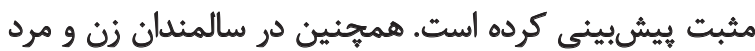

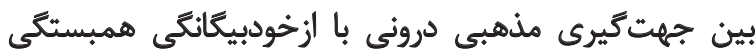

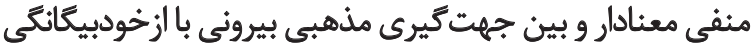

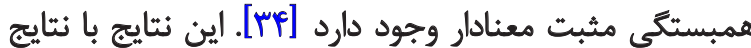

مطالعه بيشرو همسونيست مدنالت

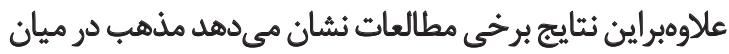

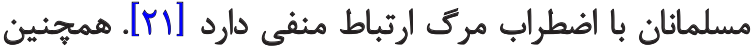

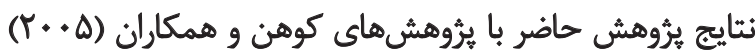

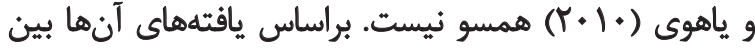

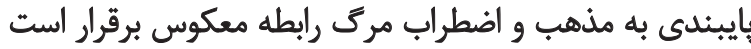

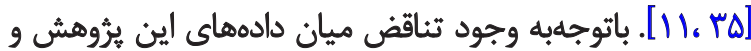

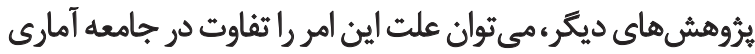

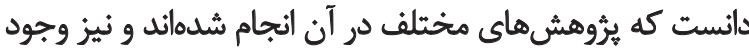

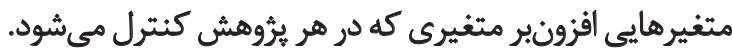
نتايج تحليل آمارى نشان داد درميان مردان مجرد و و متأهل

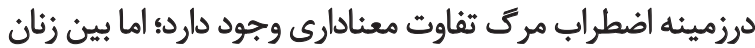

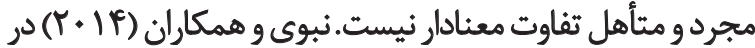

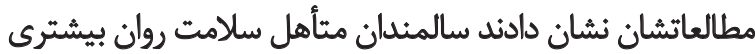

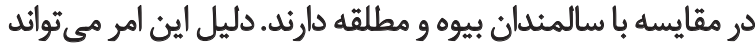

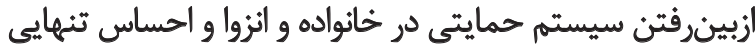

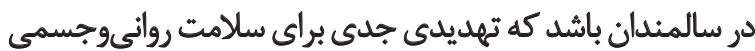

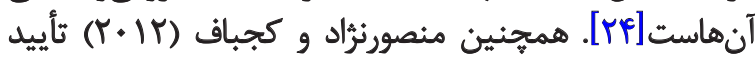

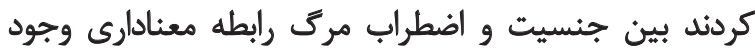

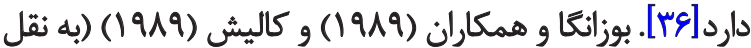

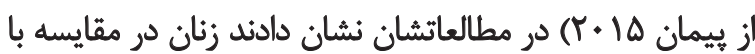

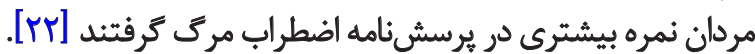

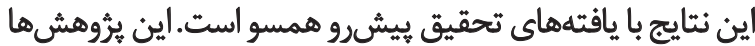

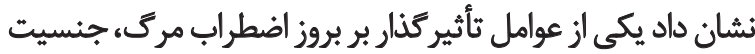

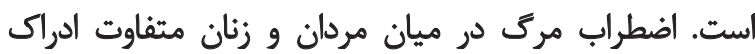

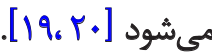

\section{نتيجه كيرى نهايي}

نتايج ثروهش حاضر نشان داد ميان حمايت اجتماعى و

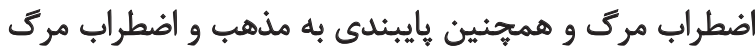
رابطه معنادارى وجود ندارد.اضطراب مركى درميان مردان متأهل مركل 


\section{References}

[1] Huerta A, Yip M. A study of religious certainty and death anxiety [Project proposal] [Internet]. 2006 [Cited 2006 Feb 17]. Available from: http://jrscience.wcp.muohio.edu/humannature06/ProposalArticles/Draft1...Astudyofreligiou.html

[2] Alipour F, Sajadi H, Foroozan A, Nabavi H, Khedmati S. [The role of social support in reducing anxiety and depression of elderly (Persian)]. Iranian Journal of Ageing. 2009; 4(11):53-61.

[3] Rashedi V, Gharib M, Rezaei M, Yazdani AA. [Social support and anxiety in the elderly of Hamedan, Iran (Persian)]. Journal of Rehabilitation. 2013; 14(2):110-15.

[4] Abdel-Chalked AM, Tomas-Sibado J. Anxiety and death anxiety in Egyptian and Spanish nursing students. Death Studies. 2005; 29(2):69-157. doi: 10.1080/07481180590906174

[5] Malliarous M, Sarafis P, Sotiriadou K, Serafeim T, Karathanasi K, Moustaka E. Greek nurses attitudes towards death. Global Journal of Health Science. 2011; 3(1):224-30. doi: 10.5539/gihs.v3n1224

[6] Geri JT. How death imitates life: Cultural influences on conceptions of death and dying. In: Loner WJ, Denel DL, Hayes SA, Sattler DN, editors. Online Readings in Psychology and Culture. Washington, D.C.: Western Washington University; 2002.

[7] Moorhead S, Johnson M, Maas M. Nursing Outcomes Classification (NOC). $3^{\text {rd }}$ ed. St. Louis: Mosby; 2004.

[8] Hoelterhoff M. Resilience against death anxiety in relationship to post-traumatic stress disorder and psychiatric comorbidity. Plymouth: University of Plymouth; 2010.

[9] Lehto RH, Stein KF. Death anxiety: An analysis of an evolving concept. Research and Theory for Nursing Practice. 2009; 23(1):2341. doi: 10.1891/1541-6577.23.1.23

[10] Vafaei Z, Asgari Zadeh GH, Rahmati A. [The relationship between sensation seeking, sense of humor and religious attitude whit death anxiety (Persian)]. Ravanshenasi-va-Din. 2011; 4(3):49-68.

[11] Cohen AB, Pierce JD, Chambers J, Meade R, Gorvine BJ, Koenig HG. Intrinsic and extrinsic religiosity, belief in the afterlife, death anxiety, and life satisfaction in young Catholics and Protestants. Journal of Research in Personality. 2005; 39(3):307-24. doi: 10.1016/j.jp. 2004.02.005

[12] Cicirelli VG. Fear of death in older adults predictions from terror management theory. Journals of Gerontology Series B: Psychological Sciences and Social Sciences. 2002; 57(4):356-58. doi: 10.1093/geronb/57.4.p358

[13] Al-Sabwah MN, Abdel-Khalek AM. Religiosity and death distress in Arabic college students. Death Studies. 2006; 30(4):365-75. doi: 10.1080/07481180600553435

[14] Suhail K, Akram S. Correlates of death anxiety in Pakistan. Death Studies. 2002; 26(1):39-50. doi: 10.1080/07481180210146

[15] Jahangirpour M, Kavand M. [The relationship between religious attitude and death anxiety among adolescences (Persian)]. Paper presented at: The $5^{\text {th }}$ Congress of Psychosomatic; 2013 May 29-31; Shahr-e Kord, Iran.

[16] Shin SH, Lee S. Aregiver social support for children involved with child welfare: Correlates and racial/ethnic variations. Journal of Public Child Welfare. 2011; 5(4):349-68. doi: 10.1080/15548732.2011.599752
[17] Salarvand Sh, Abedi H. [The elders' experiences of social support in nursing home: A qualitative study (Persian)]. Iran Journal of Nursing. 2007; 20(52):39-50.

[18] Gallangher LP, Truglio-Londrigan M. Community support, older adult's perceptions. Journal of Clinical Nursing Research. 2004; 13(1):3-23. doi: 10.1177/1054773803259466

[19] Ghufran M, Ansari S. Impact of widowhood on religiosity and death anxiety among senior citizens. Journal of the Indian Academy of Applied Psychology. 2008; 34:175-80

[20] Depaola SJ, Griffin M, Young JR, Neimeyer RA. Death anxiety and attitudes toward the elderly among older adults: The role of gender and ethnicity. Death Studies. 2003; 27(4):335-54. doi: 10.1080/07481180302904

[21] Bahrami N, Moradi M, Soleimani M, Kalantari Z, Hosseini F. [Death anxiety and its relationship with quality of life in women with cancer (Persian)]. Iran Journal of Nursing. 2013; 26(82):51-61.

[22] Peyman A. [A study of relationship between religious attitude, general health and population aspects with death anxiety in Tehran elderlies (Persian)] [MA thesis]. Tehran: Alameh Tabataba'i University; 2015.

[23] Krishna Murthy VS. Gender difference in death anxiety among HIV+/AIDS patience [PhD thesis]. Mysore: University of Mysore; 2011.

[24] Nabavi H, Alipour F, Hejazi A, Rabani E, Rashedi V. [Relationship between social support and mental health in older adults (Persian)]. Medical Journal of Mashhad University of Medical Sciences. 2014; 7(57):841-46.

[25] Tamanaeifar MR, Mansouri Nick A. [Relation between personality characteristic, social support and life satisfaction with academic performance among students (Persian)]. Institute for Research \& Planing in Higher Education. 2014; 20(1):149-166.

[26] Jahanbakhshian N, Zandipoor T. [The effectiveness of group consulting on mental health and social support based on Lazarus multidimensional approach with MS patients (Persian)]. Journal of Psychological Studies. 2011; 7(2):65-84.

[27] Seraj Zadeh H. [Attitudes and religious behaviors among Tehran adolescence and The reason for secularization theory (Persian)]. Research Index. 1998; 8(9-10):105-118.

[28] Sharifi T. [A study of relationship between religious attitude, general health, anxiety, depression, aggressiveness and patience among (Persian)] [MA thesis]. Ahvaz: Islamic Azad University; 2002

[29] Sadeghi H, Saeedi M, Rahzani K, Esfandiary A. [The relationship between social support and death anxiety in hemodialysis patients (Persian)]. Iranian Journal of Psychiatric Nursing. 2015; 2(4):36-48.

[30] Pyszczynski T, Greenberg J, Solomon S, Arndt J, Schimel J. Why do people need self-esteem? A theoretical and empirical review. Psychological Bulletin. 2004; 130(3):435-68. doi: 10.1037/00332909.130.3.435

[31] Rajaei F, Asadi A, Hasanpour E, Bahramnejad A, Fathi H, Zarkesh A, et al. [Mental health and elderly (Persian)]. Kerman: Kerman University of Medical Sciences; 2013.

[32] Nakano A. The relationship between mental health and selfrated health in older adults [Discussion paper] [Internet]. Japan: 
Kobe University; 2014 [Updated 2015 Aug 9]. Available from: http://econpapers.repec.org/paper/koewpaper/1423.htm

[33] Heine C, Browning CJ. Mental health and dual sensory loss in older adults: a systematic review. Frontiers in Aging Neuroscience. 2014; 14(6):83-6. doi: 10.3389/fnagi.2014.00083

[34] Ali Akbari Dehkordi M, Oraki M, Barghi Irani Z. [Relation between religious orientation with anxiety about death, alienation in aged peoples (seniors) in Tehran (Persian)]. Social Psychology Research Quarterly. 2011; 1(2):140-150.

[35] Wen YH. Religiosity and death anxiety. Journal of Human Resource and Adult Learning. 2010: 6(2):31-37.

[36] Mansournejad Z, Kajbaf M. [The relationship of religious orientation (intrinsic, extrinsic) and gender with death anxiety among students (Persian)]. Cognitive and Behavioral Research. 2012; 2(1):55-64. 
